\title{
Manipulations and Spins in Attention Disorders Research: The Case of ADHD and COVID-19
}

\author{
Yaakov Ophir, $\mathbf{P h D}^{1}$ and Yaffa Shir Raz, $\mathbf{P h D}^{2,3}$ \\ ${ }^{1}$ Technion - Israel Institute of Technology, ${ }^{2}$ The Interdisciplinary Center Herzliya, ${ }^{3}$ University of Haifa \\ *Correspondence: Yaakov Ophir, E-mail: yaakov.ophir@mail.huji.ac.il.
}

\begin{abstract}
Background: Critics of Attention Deficit/Hyperactivity Disorder (ADHD) warn against overdiagnosis and overtreatment of millions of children. Objective: The goal of this article is to raise awareness to manipulations and "spins" that might occur in attention disorders research. Method: An in-depth inspection was conducted on a high-profile study that suggested that ADHD increases the risk for infection with COVID-19 and that stimulants reduce that risk ${ }^{1}$. Two additional studies by the same first author were inspected as well - one that was published in the same journal and one that relied on the same dataset. Results: Seven manipulations and spins were identified, including inappropriate operational definitions, misrepresentations, and omissions that produced bogus results and might have concealed potential adverse effects of medications on COVID-19 outcomes. Without correcting for these distortions, it is therefore not possible to determine that ADHD increases the risk of COVID-19 and that stimulant medications reduce this risk. Conclusions: The manipulations and spins identified in this article may raise awareness to poor methodologies and biases that occur in the field of attention disorders, mainly due to the contaminating influences of the pharmaceutical industry. In turn, these biases may contribute to the ethically problematic phenomena of overdiagnosis and overmedication. Altogether, this article may encourage physicians to apply healthy scientific skepticism and serve scientists who wish to conduct reliable and transparent research on ADHD and its related medications.
\end{abstract}




\section{Introduction}

A tention Deficit/Hyperactivity Disorder (ADHD) and its associated medication treatments stand at the heart of a long-standing controversy in the medical, legal, and ethical discourse $\mathrm{e}^{2-4}$. The intensity of the controversy is fueled, among other reasons, by the fact that the diagnosis of ADHD is far more prevalent among underaged children than in adult populations ${ }^{5}$. On the one hand, several scholars argue that the symptoms and etiology of the disorder suffer from poor scientific validity ${ }^{6}$ and warn against over-diagnosis and overmedication (Mayes, Bagwell, \& Erkulwater, 2008; Merten, Cwik, Margraf, \& Schneider, 2017) of millions of children ${ }^{7,8}$. On the other hand, the scientific consensus is that ADHD is a well-documented, neurodevelopmental condition ${ }^{9,10}$, with numerous negative outcomes ${ }^{11-13}$. ADHD, according to this dominant view, often persists into adulthood and should therefore be carefully managed throughout the person's life $^{9,11}$. In most cases, this management involves the usage of psychostimulant medications, such as methylphenidate, amphetamines, and methamphetamines ${ }^{7,8,14}$, the first-line pharmacological treatment for $\mathrm{ADHD}^{15,16}$.

Despite their documented short-term benefits ${ }^{17}$, many people do not adhere to their prescribed stimulant medications, due to their adverse side effects ${ }^{18}$. In order to increase patients' compliance to pharmacological treatments, two complementary lines of research are promoted by members of the scientific consensus: studies that demonstrate the potential negative outcomes of ADHD, and studies that show the beneficial effects of ADHD medications in reducing these negative outcomes.
The potential negative outcomes of ADHD are easily communicated. The core symptoms of ADHD hyperactivity, impulsivity, and poor cognitive inhibitions - may result in a range of adverse outcomes ${ }^{19}$. Individuals with ADHD tend to take more risks ${ }^{20}$ and engage in highly stimulating behaviors, such as reckless driving, unprotected sex, as well as extreme sports and outdoor activities. These risk taking behaviors may result in: physical injuries ${ }^{21}$, car accidents $^{22}$, substance abuse ${ }^{23}$, and sexually transmitted diseases $^{24}$. Similarly, difficulties in cognitive and emotional control ${ }^{12}$ may also result in criminal behaviors $^{25}$, incarceration ${ }^{26}$, suicide attempts ${ }^{27}$ and even early mortality ${ }^{28}$.

Complementing this line of research, multiple studies on ADHD medications were conducted to demonstrate their potential positive effects in moderating this long list of risks. An updated literature review and meta-analysis concluded that ADHD medications may serve as a protective factor against a wide range of ADHD-associated negative outcomes ${ }^{19}$. Medications use has been documented to decrease the risk for accidents and injuries ${ }^{29}$, criminality $^{26}$, substance abuse $^{30}$, and suicidality ${ }^{31}$.

Taken together, these findings - regarding the potential negative outcomes of ADHD and the potential positive effects of ADHD medications - point to one conclusion: Individuals with ADHD should adhere to their prescribed medications because otherwise they increase their chances to suffer from the numerous negative outcomes of $\mathrm{ADHD}^{17}$.

This conclusion seems highly reasonable, assuming that the two complementary lines of research are based on objective and reliable studies. However, transparency and objectivity are not always applied in bio-medical 
research. A recent literature review published in the US National Academy of Sciences journal (PNAS) revealed a list of distortions, manipulations and spins, that might occur in bio-medical research ${ }^{32}$. The concept of "spin" has been borrowed from popular/political discourse to describe intentional or unintentional scientific practices, which are applied to a given dataset, in order to achieve or present favorable results, without actually lying ${ }^{32}$. These practices include dozens of inappropriate methodological and representational decisions, such as undisclosed flexibility in the data collection and the applied analyses ${ }^{33}$, selective reporting of outcomes, and omissions of adverse events ${ }^{34}$.

Manipulation and spins, as mentioned above, are not necessarily intentional. Scientists from all fields of knowledge, including ourselves, may sometimes temporarily forget the principal goal of science - to discover truths, and unintentionally make unjustified methodological decisions, in order to solve ambiguities and achieve publishable outcomes ${ }^{33}$. Yet, the specific research field that addresses attention disorders seems highly vulnerable to manipulations and spins, due to the close collaborations between mental health scientists and pharmaceutical companies ${ }^{35}$. The pharmacological treatments for ADHD rank at the top of the best-selling drugs for children ${ }^{36,37}$, and numerous ADHD researchers maintain relationships with pharmaceutical companies ${ }^{18}$. Correspondingly, a recent review by the Cochrane organization rated the vast majority of the 260 reviewed studies on ADHD medications as studies with very poor scientific quality and with a critical risk (the highest possible risk) for bias ${ }^{18}$.

Of course, poor scientific quality and inherent conflicts of interests do not necessarily mean that all the studies on ADHD produce unreliable findings. Yet, they do require an extremely careful reading and interpretation. The goal of the current paper is to raise awareness to illegitimate manipulations and spins that might occur in attention disorders research, by carefully examining a recent high-profile study titled: "ADHD as a Risk Factor for Infection With COVID-19"1. We focus on this research as a case study because it provides a condensed and vivid illustration for a multilayered set of manipulations and spins, designed (consciously or unconsciously) to produce desirable conclusions, without actually lying.

On July 22, 2020, in the midst of the COVID-19 crisis, Merzon and colleagues (2020) published a troubling article, in which they warn that "ADHD may be a risk factor for COVID-19 infection, independently of other risk factors". This conclusion seemed, at first glance, highly reliable, as it was extracted from objective medical records of 14,022 individuals who underwent a test for COVID-19 during the 3-monthes period (February 1 - April 30, 2020) of the first Corona wave in Israel. It is no surprise then, that it received considerable public attention. For the first time, to our knowledge, ADHD was linked to a highly infectious global epidemic, perhaps reflecting an escalating new trend in attention disorders research. Complementing this warning, the second conclusion of the study comprised 'a call for action'. Because pharmacotherapy was found in the study to ameliorate the risk for infection and play a potential protective role "in the attenuation of COVID-19 transmission" (page 6), the authors advise individuals with ADHD to adhere to their prescribed stimulant medications ${ }^{1}$.

The current article questions the validity of Merzon, Manor et al.'s conclusions. Are individuals diagnosed with ADHD really at increased risk for 
COVID-19? Are they indeed unknowingly contributing to the spread of the dangerous virus? Should parents of children diagnosed with ADHD hurry to give them stimulant medications to protect them against the virus or to attenuate its spread? To address these questions, we examined the study on ADHD and COVID-19 by carefully tracking the authors' methodological decisions and by comparing these decisions to two additional studies that were published by the first author at the same week, one of which was published in the same journal $^{38}$ and another one, which relied on the same dataset, was published in the FEBS Journal ${ }^{39}$.

This investigation revealed multiple problematic methodological and representational decisions that challenge the validity of the authors' conclusion that ADHD increases the risk for COVID-19 infections and undermine their recommendation to consume stimulant medications. It is our hope that the manipulations and spins found in our inspection will encourage practitioners to apply scientific skepticism when encountering similar intimidating titles and serve fellow scientists who wish to conduct reliable and transparent studies on the ADHD and its related medications.

\section{In depth inspection of the study on ADHD and COVID-19}

After its publication in the Journal of Attention Disorder, the study on ADHD and COVID-19 received considerable media attention, due to its timely topic and thought-provoking conclusions. Almost at the same time, the first author published two additional studies, which also relied on Leumit medical records. One of these studies was actually conducted on the very same dataset. Intrigued by the public discourse that was triggered by the publication of the ADHD and COVID19 study, we read all three studies, with great interest.
However, while we truly appreciated the efforts that were invested in these studies, we also encountered several issues, which required further understanding. We then issued the corresponding author and the first author of the study, two separate requests (one on July 29, 2020 and one on August 5, 2020), to receive access to the dataset. These requests were sent through a neutral party (i.e., a researcher that is not involved in the debate regarding ADHD). Nonetheless, to date (August 19, 2020, three weeks following our initial contact), we were not granted this access, despite the explicit declaration that all data "can be accessed following approval of Leumit health services" (page 6). Due to the importance of this topic, especially in these complex and sensitive times of COVID-19 pandemic, we decided to examine the methodological and representational decisions that were taken in the study, as well as the two additional studies by the same first author. This process yielded seven problematic and illegitimate practices that can be categorized into two types: (A) manipulations of operational definitions and (B) spins of the data and the results.

\section{A. Manipulations of operational definitions}

The inspection of the manuscript yielded problematic manipulations of three operational definitions - the definition of ADHD, the definition of ADHD medications, and the usage of inappropriate age categories.

1. Unreliable and inconsistent definition of ADHD. A first manipulation was observed in the definition of the primary variable of the study (i.e., the variable of ADHD). Based on the Leumit medical records, participants in the study at hand ${ }^{1}$ were defined as 'individuals with ADHD' if they had ever received a diagnosis of ADHD. This might seem as a reasonable 
methodological decision, however, two of the authors (the first and the sixth ones, together with other colleges) used a different operational definition in their study on childhood shigellosis and $\mathrm{ADHD}^{38}$. Unlike the study at the center of this paper, in this other study, ADHD was determined only if the participants (1) had a diagnosis of ADHD and (2) had purchased at least three prescriptions for ADHD medications. This twist is particularly surprising in light of the fact that the study on shigellosis was published only one day later (July 23, 2020) in the same journal (Journal of Attention Disorders). Moreover, both studies relied on the same source of data - the highly reliable Leumit medical records. So why would one study adhere to two criteria - both "having a diagnosis of ADHD" and "purchasing at least three prescriptions", while the other one settles for just the first criterion?

Interestingly, the authors explain that they applied this second criterion (and excluded diagnosed individuals who did not purchase three prescriptions) because ADHD, is "given temporally with referral of the patient to ADHD clinic"38. In other words, a diagnosis of ADHD per se, according to the authors themselves, is an unreliable operational definition for the existence of ADHD. Why then do two of the authors of the shigellosis study base the definition of ADHD solely on this unreliable definition in their study on Coivd-19? This inconsistency of definitions, along with the possible use of an unreliable definition for ADHD, raise the possibility that different operational definitions were chosen for each study, to achieve desirable results.

Aside from the proximity in time and the fact that both studies relied on Leumit medical records, a strong indication that the operational definitions of ADHD were manipulated is the substantial differences in
ADHD rates that were reported in each study. While Merzon, Gutbir, et al., reported of ADHD rates that ranged from $8.6 \%$ to $10.6 \%$ among children aged 5-18 years (based on their 2-creterions operational definition for ADHD), Merzon, Manor, et al., reported of ADHD rates that ranged from of $18.85 \%-28.14 \%$, among individuals aged 5-20 years. Based on the information that was provided, we calculated that $20.25 \%^{1}$ of young individuals (5-20) received a formal diagnosis of ADHD. This is a huge gap that should not be hidden from the readers of the Journal of Attention Disorders. Moreover, not only that this exceptionally high prevalence is not consistent with the authors' own findings, it is also inconsistent with the documented prevalence of the disorder in the literature, as manifested in other conventional sources, such as the Centers for Disease Control and Prevention (CDC) survey $^{7}$ and the Diagnostic and Statistical Manual of Mental Disorders (DSM) estimates ${ }^{5}$. This last consensual source (DSM) for example, provides an estimation, according to which $5 \%$ of all children suffer from ADHD.

Discrepancies between a given study and its field of research is a well-recognized problematic phenomenon $^{40-42}$. However, here we evidenced a unique discrepancy. Not only that the observed rates of ADHD were not consistent with conventional rates in the literature, they were not even consistent with the authors' own reports from a similar dataset (Leumit medical records). In fact, the deviant high prevalence of ADHD (20.25\%) confirms the concerns that were raised by the authors of the shigellosis study ${ }^{38}$ regarding the reliability of the diagnosis (i.e., that the diagnosis is

\footnotetext{
${ }^{1}$ This figure (the total prevalence of ADHD among young individuals) is not explicitly reported in the article but could be calculated from the available data.
} 
given temporarily). In other words, it is possible, according to two of the authors, that a large percentage of the participants in the study on ADHD and COVID19 is misdiagnosed. So, unless ADHD became the new pandemic, it is imperative to consistently adhere to the authors' more cautious operational definition of ADHD (a diagnosis of ADHD + three prescriptions). Interestingly, by applying this more cautious operational definition of ADHD, which includes the participants' usage of medications (see also the next point), we might conclude that there is no evidence that confirmed ADHD increases the risk for COVID-19 infections.

\section{Inappropriate definition of ADHD} medications. Another manipulation was identified in the secondary variable of the study - medication treatments. The authors define medication treatments by documenting those "who purchased at least three consecutive prescriptions of ADHD medications during the past 12 months" (page 2). This raises two problems: First, this operational definition (or a similar one), could have been used to define the very existence of ADHD (point 1), which would have altered the results altogether. Second, and no less problematic, the '12 months' criterion is unjustified. What could be the justification to include prescriptions from the past 12 months, while the outcome measure (positive for COVID-19) is confined to a 3-months period of the first Corona wave in Israel? The calming effects of stimulant medications on ADHD symptoms (which are speculated by the authors to increase COVID-19 infections) last between 4-12 hours ${ }^{43}$ and once the effects fade out, the symptoms usually return (some users may even experience a 'rebound effect'). To our knowledge, even the proponents of the medications do not assume that past usage of stimulant medications (e.g., 9-11 months ago) can be relied on for current management of ADHD symptoms. On the contrary, the European scientific consensus (which includes the corresponding author of the study at hand) seems to advocate the position according to which ADHD is a neurodevelopmental lifelong condition, which requires a constant management ${ }^{9}$, and most scholars agree that the longterm effects of stimulant medications are yet to be studied $^{17,44}$.

Therefore, there was no reason for the authors of the study at hand to assume that medications taken several months before the disruption of the Corona virus, could have reduced the person's current behaviors that led to his/her COVID-19 infection. A much more reasonable methodological decision would be to define 'treated individuals' according to their current usage of medication (e.g., purchasing prescription medications during the confined 3-months period of the study, or a month before that). Another indication that the firm cutoff point that was eventually chosen (three consecutive prescriptions in one year) is an illegitimate manipulation, is the fact that it resulted in very low rates of medication use (24.6\%), compared with other, well cited sources $(62 \%-76 \%)^{7,8,45}$. In other words, the data that was available to the authors on medication use was reduced in an arbitrary manner ${ }^{34}$. We therefore carefully raise the question: was this operational definition tailored specifically to bias the results?

\section{Arbitrary and irrelevant age categories. A} third major inappropriate manipulation of definitions was identified in the central variable of age. Besides the fact that age is a continuous, and not a categorical variable, the chosen categories $(\leq 20,21-40,41-60$, and $>60$ ) were arbitrary and irrelevant to the subject of the study (ADHD). Specifically, the manipulation pertains to the first category. Why was the age of 20 chosen as a 
cutoff point and not the age of 18, which is the conventional cutoff point for adulthood, and more importantly - why did the authors disregard crucial age differences within this age group? What is the justification to include a 5-year-old boy together with a 16-year-old teenager, let alone a 20-year-old young adult?

Even if we overlook the inappropriate definitions of ADHD and of medication treatments (points 1 and 2), the decision to ignore age differences among young participants undermines the very conclusion the study. The diagnosis of ADHD, as well documented in the literature, is significantly more prevalent among children and adolescents, compared with adult populations ${ }^{5,7}$. This known fact is also evident in the study at hand: individuals with ADHD were significantly younger (mean age $=25.31$ ) than the rest of the sample (mean age $=40.82)^{2}$. In order to prove that ADHD serves as a risk factor for COVID-19, above and beyond the potential effects of age, researchers should have investigated the age differences within this young, and highly diagnosed, group.

Differentiating between the effects of age and ADHD is especially important in light of the close and intertwined relationships between 'age' and the diagnosis of ADHD. ADHD can only be diagnosed if the symptoms (e.g., often runs about, often leaves seat) are not explained by the individual's developmental stage. In fact, some children may be misdiagnosed only because they are younger than their classmates ${ }^{46-48}$. Moreover, during natural development, hyperactive behaviors tend to decrease and cognitive abilities are inclined to improve ${ }^{49}$ and many children will not be

${ }^{2}$ The mean ages in the parentheses are not explicitly reported in the article but could be calculated from the available data. eligible for the diagnosis when they become adults ${ }^{5}$. Even brain differences between diagnosed and nondiagnosed children disappear in adult populations ${ }^{50}$.

The decision to disregard age differences in the first age group $(\leq 20)$ is problematic not only from a theoretical point of view - it does not fit the specific dataset of the study, in which the most dominant risk factor for COVID-19 infection was young age $(\leq 20)$ (adjusted OR $=2.08$ ). In fact, the risk among young individuals was $50 \%$ higher than the alleged risk among individuals with ADHD (adjusted OR = 1.58). Unless the authors recommend to all young individuals to use stimulant medications (which were previously argued to protect from COVID-19), this most powerful finding should be further investigated. Playful young children who engage in behaviors that might increase the risk for COVID-19 (e.g., run about, leave their seats), cannot be aggregated with adolescents or young adults, who have more behavioral constraints and a deeper understanding of the concept of social distancing. Therefore, if we truly wish to identify the risk of ADHD beyond the potential effect of age, we have to create relevant categorical subgroups (e.g., preschool, elementary school, middle school, high school, and $\geq 18$ ). Only by properly considering the effect of age, one can assess the relationship between ADHD and COVID-19.

\section{B. Spins in representations of data and results}

In addition to the inappropriate definitions of the variables, which severely impaired the reliability of the findings, narrative spins were identified in the presentations of the results, both in the abstract and in the body of the study itself.

4. Misrepresentations of the results. While the results regarding ADHD are presented in the abstract using an Adjusted OR score (as they should), the results 
regarding the protective role of medications are presented (only one line below) using a Crude OR score $(1.07, \mathrm{p}=.65)$. This twist is surprising for two reasons: First, the Crude OR statistic is less accurate than the Adjusted OR. Second, the Adjusted OR (0.85, $p=.707)$, which can be found in table 4 , is far more impressive, from the authors' point of view, as it drops below 1 (thus implying of a potential protective factor).

Moreover, in the results section, the authors report of an even more impressive finding regarding the potential role of medications - a significant and very low adjusted OR score of 0.63 ( $\mathrm{p}<.001)$. We assume that this last figure represents a comparison between treated and untreated individuals with ADHD, rather than a comparison with the entire sample (including individuals without ADHD), which might be less relevant to the main narrative of the study. Surprisingly, this impressive finding, which one would expect to be highlighted in the study, is not explicitly mentioned in any other sections, neither in Table 4 or Figure 1, nor in the abstract. These odd and inconsistent presentation choices raise the suspicion of a narrative spin in the framework of the article. Why would the authors miss the opportunity to highlight this remarkable finding? Also, if the authors prefer to remain with the comparison among the entire sample (and not just among the ADHD group), why not present the adjusted OR of 0.85 . Is it because this positive finding was not significant, thus suggesting that medications are not proven to protect from COVID-19?

\section{Omission of available data on adverse} outcomes of COVID-19. Misreports were also observed in the authors description of the outcome variables of the study. A major weakness of the study, according to the authors themselves, is the fact that "data regarding the presenting symptoms and severity of COVID-19 infection, as well as adverse clinical outcomes (hospitalization and mechanical ventilation) were not assessed"1. This use of the words "not assessed" implies of a spin in light of the fact that the authors had access to the health records of Leumit, which according to their own description included "demographics, medical visits, laboratory tests, hospitalizations, and medication prescriptions" (page 2). Moreover, in a related study on vitamin D and COVID19, which apparently examined the very same dataset of 14,022 Leumit medical records, the first author, together with other researchers ${ }^{39}$, did assess the adverse outcomes of COVID-19. Like the study on shigellosis, this study on vitamin D was published only one day after the publication of the COVID-19 research (July 23,2020 ) in the FEBS Journal, thus suggesting that the authors did assess the adverse outcomes of COVID-19. Nevertheless, they did not report these outcomes in their study on ADHD and COVID-19.

This decision, to disregard data available to them on severe outcomes of COVID-19 is disturbing for two reasons: First, COVID-19 is mainly dangerous to old populations, while children rarely exhibit severe symptoms ${ }^{51}$. This means that the inclusion of severe outcomes of COVID-19 in the study could have proven that the ADHD group (which is significantly younger than the rest of the sample) is in fact the safest group to be in, with very little or zero number of hospitalizations and ventilations (assuming of course that the authors adhere to their inappropriate age category that does not distinguish children from adolescents and young adults, which was described in point 3 ). This potential finding is of course not in line with the authors' conclusions, thus raising the possibility that the omission of adverse outcomes, which were available to the authors, is a spin. 
Second, if ADHD is indeed not a risk factor for severe outcomes of COVID-19, then the recommendation to parents to keep administering stimulant medications to their children, even when schools are closed (due to the pandemic lockdown), is ethically problematic. Are we really aiming at prescribing medications for the hypothetical greater good of others and not for the benefit of the consumers of the medications themselves? In the cost-benefit considerations (that should characterize the usage of any medical treatment), the risk for adverse outcomes of COVID-19 for children is negligible, while the risk for adverse outcomes of stimulant medications is wellestablished. Most children who use stimulant medications exhibit at least one mild adverse side effect and about $1 \%$ exhibit dangerous or severe adverse effect $^{18}$ (for further information, see the discussion section).

\section{Selective data analyses and presentations (in}

two different articles). A further comparison between the two studies by the first author, the one in the FEBS Journal and the one in the Journal of Attention Disorders, raises the speculation that selective data was reported in each study to achieve desirable results. In the FEBS Journal, the authors report of a finding which has important potential implications for the public health. According to the authors, levels of vitamin D may serve as a protective factor against adverse effects of COVID-19. Considering this fascinating finding and the fact that both studies analyzed the same dataset, authors of the ADHD study ${ }^{1}$ should have controlled for the promising effect of vitamin D. Without this statistical control, the exact risk of ADHD (i.e., the Adjusted OR) is inaccurate. But more importantly, without controlling for vitamin $\mathrm{D}$, the authors deprive readers of vital information that could have helped them decide whether to medicate their children or simply expose them to more sunlight and outdoor activities ${ }^{52}$.

On the other side of this equation, authors of the vitamin D study should have controlled for the effects of ADHD. In fact, in this last study on vitamin D, the authors do control for a variety of psychiatric and somatic disorders, but for some reason, they disregard the ADHD diagnosis, which, as we already know, was available to them. Why would the authors of the vitamin D study ignore this specific diagnosis? Is it because they evidenced results that did not match the narrative that was told in their equivalent study on ADHD?

\section{Omission of potential effects of medications} on adverse outcomes of COVID-19. Finally, a spin might have been applied to conceal the potential adverse side effects of the medications ${ }^{34}$. For some reason, the authors of the vitamin D study did not address the effects of ADHD medications on severe outcomes of COVID-19. This is despite the fact that the first author probably had access to this information, as manifested in the data description that is provided in his study on ADHD (p. 2). Without having access to the raw data, it is hard to judge the severity of this omission and to estimate whether it conceals the potential role of stimulant medications in the development of severe COVID-19 outcomes. Yet, this situation raises the concern that non-scientific interests might have contaminated the presentation of the findings from both articles.

Stimulant medications are known to have potential negative cardiovascular consequences ${ }^{53}$, and may therefore be unsafe to use during the COVID-19 pandemic. Correspondingly, the newly released European Guidelines on starting ADHD medications during the COVID-19 pandemic ${ }^{54}$ determine that 
ADHD medications should not be started if the person has a history of breathing and/or cardiovascular-related problems (e.g., fainting or shortness of breath on exertion; excessive palpitations; chest pains; heart abnormalities, previous cardiac surgery, or underlying condition that increases the risk of cardiac disorder). Physician should also not prescribe ADHD medications to individuals who have a family history of early $(<40$ years) sudden deaths. Moreover, psychostimulants (as learned from studies on neurotoxins, such as methamphetamines) might alter the blood-brain barrier permeability and thus increase the risk for invasion of dangerous viruses into the brain ${ }^{55,56}$. In light of these warnings, as well as the known side effects of stimulant medications, the authors should have ruled out the possibility that the medications are not safe for individuals infected with the COVID-19.

Not only did the authors not report/analyze the severe outcomes of COVID-19 (see also point 5), they actually cite the above European guidelines as if they were proof to their own recommendation, according to which "Adherence to anti-ADHD treatment should be encouraged in an attempt to reduce the spread of COVID-19 infection" (Cortese et al., 2020). This is a noteworthy spin, because the guidelines suggest the opposite - that ADHD medications may not be appropriate in times of COVID-19, because of their potential adverse outcomes. We therefore assert that the allegedly protective role of the medications against COVID-19 could only be properly evaluated by analyzing the medication effects on the adverse outcomes of the virus. The omission of such outcomes (e.g., hospitalization, mechanical ventilation), as discussed above (point 5), raises the concern that critical information regarding the medications was hidden from the readers. Can stimulant medications worsen the symptoms of COVID-19?

\section{Summary and discussion}

In this article we have carefully examined a recent publication in the Journal of Attention Disorders, in which the authors argued that ADHD is a risk factor for COVID-19, independently of other risk factors (such as age), and that stimulant medications may have a protective role "in the attenuation of COVID-19 transmission"1. This investigation identified three manipulations of variables and four spins of data and results that challenge the validity of the authors' serious claims. These included: (1) Unreliable and inconsistent definition for ADHD, (2) Inappropriate definition for ADHD medications, (3) Arbitrary and irrelevant age categories, (4) Misrepresentations of the results (5) Omission of available data on adverse outcomes of COVID-19, (6) Selective data analyses and presentation in two different articles, and (7) Omission of potential effects of medications on adverse outcomes of COVID19.

In light of the list of spins and manipulations identified in this investigation, the authors' conclusions that ADHD increases the risk of COVID-19, and that stimulant medications reduce this risk, cannot be confirmed. On the contrary, the multiple unjustified methodological and representational decisions raise the uncomfortable feeling that manipulations and spins were applied to the dataset in order to achieve desirable (bogus) results and conceal unwanted findings.

Such manipulations, and spins are not new to the scientific community, especially in the bio-medical field $^{33,57}$. Previous studies have shown similar distortions and misrepresentations, including: Ignoring contradicting results ${ }^{32}$; Reducing data in a biased 
fashion; Ignoring adverse outcomes, Inappropriate subgroup analyses; and Selective reporting of subgroup and outcomes ${ }^{34}$. Earlier studies also warned against the potential ramifications of such distortions and their biased impact on clinicians and patients ${ }^{58,59}$, and suggested valuable scientific practices that could reduce these biases (e.g., register and share datasets with the scientific community $)^{60,61}$. However, the convergence of seven blunt distortions in one study along with the fact that the authors avoided granting access to the dataset, call for a renewed public and scientific discussion. Such a discussion is especially essential in light of the potential large impact of the study on the decisions of many parents, and hence on the lives of numerous children, in these complex and sensitive times of COVID-19.

As noted above, the problem of scientific misconducts and distortions may exist in all fields of research $^{33}$. However, the field of attention disorders is particularly vulnerable to such malpractices. This field has long been criticized for over-diagnosis and overmedication of millions of children (Mayes, Bagwell, \& Erkulwater, 2008; Merten, Cwik, Margraf, \& Schneider, 2017), and for being dependent and highly influenced by the pharmaceutical industry ${ }^{35,62}$. Indeed, in the study on ADHD and COVID-19, the authors declared no potential conflict of interests and we assume that this was indeed the case. However, given the long list of manipulations and spins, which happened to corroborate with the interests of pharmaceutical companies, one cannot help but wonder whether the study has been driven by non-purely scientific interests. Specific economic interests might have been evoked during the COVID-19 crisis. ADHD medications, as described above, are among the best-selling drugs for children ${ }^{36,37}$ but during the first wave of the pandemic, medications sales might have dropped significantly. In a small scale study $(N=136)$, which we have conducted recently, we found that approximately $70 \%$ of mothers whose children take ADHD medications, instructed their children to stop using them during the first COVID-19 wave lockdown ${ }^{63}$.

We trust that the authors of the study examined in this paper did not have explicit conflict of interests, as they reported. However, it is noted that the pharma industry consistently target their marketing efforts at physicians and researchers by providing them research grants, traveling expanses, and personal gifts ${ }^{35,62,64}$. An updated research that tracked the marketing expanses on ADHD medications revealed that between 2014-2018, the pharma companies have transferred over than 20 million dollars' worth gifts and meals to 55,105 pediatricians, psychiatrists, and family physicians ${ }^{65}$. Most physicians, we assume, only prescribe ADHD medications, when they believe it will improve their patients' lives. Yet, by the end of the day, meals and gifts that are sponsored by a given medication brand are quickly translating into a rise in the prescriptions of the brand $^{66}$.

In the same way, pharmaceutical companies' money may spoil the objectivity that is required for scientific research. A recent Cochrane review found that approximately $30 \%$ of non-comparative cohort studies were funded directly by pharmaceutical companies and more than 5\% included authors who had relationships with pharmaceutical advisory boards ${ }^{18}$. Disturbingly, the studies that were funded by the industry reported of significantly less serious and non-serious adverse side effects of the medications, compared with non-funded studies. Inevitably this phenomenon leads to bad science. As mentioned above, almost all 260 studies 
reviewed by the Cochrane were rated with very poor scientific quality and with the highest risk for bias ${ }^{18}$.

Studies at risk for bias, which warn against potential adverse outcomes of ADHD while emphasizing the benefits of medications, should therefore be carefully interpreted. Similar to the study at the center of this paper, other studies in this field may exhibit similar shortcomings. The updated meta-analysis presented in the introduction ${ }^{19}$ for example, ends with the following statement: "All authors report no potential conflicts of interest to disclose above the ones reported in the financial disclosures". Apparently, these financial disclosures (which were only displayed after the long list of references) included conflicting interests that might have infected the meta-analysis. These interests may explain the observed gap between the conclusion of the meta-analysis and its' actual findings. The abstract of the meta-analysis concluded that the findings "suggest that ADHD medication treatments are associated with decreases in the risks for a wide range of ADHD-associated functional outcomes" (page 21). Yet, the picture that arises from the findings is in fact very different. The overall "protective" effects of medications on suicidality and on traumatic brain injury relied on two studies only, suffered from significant heterogeneity and most importantly, were not significant. The overall effects on substance use and on criminality also relied on two studies and were also not significant. Finally, the only potential significant overall effect on accidents and injuries that relied on several studies $(N=6)$, suffered from significant heterogeneity, which challenge the interpretation of the effect. Surprisingly, even the effects on academic outcomes, as measured by three studies with continuous dependent variables (i.e., not categorical variables that allow the extraction of an OR score) were not consistent. In fact, the overall effect on academic outcomes was neutral. A plausible explanation for this unexpected finding is that it resulted from the fact that the studies were highly heterogeneous and produced positive, neutral, and even negative findings (i.e., the medications were also shown to have negative effects on academic performance).

Moreover, although the authors of the metaanalysis specifically stated that they "used the Egger method to assess for publication biases" (page 22), they only provide this crucial information in their last analysis on academic performance (which, as mentioned above, produced a natural overall effect). Why would the authors choose to omit such basic information? Is this choice related to their relationships with the pharma companies? When examined together - the relationships with the pharma industry, the omissions of publications bias results, and the mostly non-significant, unreplicated and heterogeneous overall effects of the medications - challenge the conclusion of the metaanalysis, as if ADHD medications can decrease the risks for "a wide range of ADHD-associated functional outcomes".

Whether intentional or not, methodological manipulations and spins are especially dangerous in the case of ADHD medications. Indeed, many ADHD experts believe that stimulant medications "are relatively safe", at least for the short-term ${ }^{16}$ but a the systematic review that was mentioned above found that most children exhibit at least one mild adverse side effect of stimulant use ${ }^{18}$, including for example, anxiety, dizziness, irritability, nightmares, restlessness, sadness, and tics ${ }^{67}$. About $1 \%$ (which is a large number considering the millions of children who use these medications) exhibit dangerous or severe adverse effect $^{18}$. A short list of these serious effects include: 
cardiovascular disruptions ${ }^{53,68-71}$, psychotic or manic episodes $^{72-76}$, and even premature death ${ }^{77,78}$.

Not only do stimulant medications have multiple adverse side effects, they are given mainly to underage children $^{36,37}$. Children trust their caregivers to provide them the best care they can, and caregivers trust us, scientists and practitioners, to provide them reliable and uninfected scientific information. It is our view that without proper scientific grounding, claims regarding the dangerous nature of ADHD and the benefits of medications, which are followed by an explicit recommendation to adhere to prescribed medications do not meet the basic ethical guidelines of medical treatments. Most parents do not have the means to track the multiple manipulations and spins that occur in the studies that advocate the benefits and safety of ADHD medications and might believe their take-homemessages that are broadcasted over mass media platforms. They might even believe the unfounded and provocative claim that ADHD increases the risk for COVID-19, and rush to comply with the experts' recommendation to use unsafe stimulant medications, even when schools are closed.

The validity of the identified distortions is somewhat limited, due to our lack of access to the raw data. Therefore, we cannot rule out the possibility that the authors could have achieved similar results, even after implementing all the necessary corrections in the dataset. However, the fact that some of the distortions involved blunt and unexplainable gaps between the authors' own reports lend strong support to the refutation that was presented in the current paper. A second limitation concerns the generalizability of the manipulations and spins that were identified in this paper. It is possible that the multiple manipulations and spins that converged in this study on ADHD and COVID-19 are not representative of other studies in the field of attention disorders. Nevertheless, it is noted that the bio-medical literature consists of many misconducts and distortions ${ }^{32,34,57}$, and the specific field of attention disorders is highly susceptible to such distortions, due to the poor scientific quality and critical risk of bias that characterize many of the studies on ADHD medications ${ }^{18}$, which in many cases result from the contaminating influence of the pharmaceutical industry ${ }^{35}$.

In fact, Dr. Keith Conners himself, who is sometimes referred to as the "father of ADHD" warned that the disorder has unjustifiably became an "epidemic", to allow the prescriptions of unprecedented numbers of medications ${ }^{62}$. The current article raises the concern that this "epidemic" of overdiagnosis and overmedication is fueled by poor quality and biased science. It is our hope that the distortions that were identified in this article will empower parents, as well as physicians, to apply healthy scientific skepticism when encountering similar titles that warn against the harms of ADHD. We also hope that the detailed list of manipulations and spins provided in this article will help readers identify other questionable studies in attention disorders research and serve fellow scientists who wish to conduct reliable, valid, and most importantly, transparent studies on ADHD and stimulant medications.

\section{Declaration of interests}

The authors declare no conflict of interests. The authors did not receive financial support for this article or to any other activity they had done in relation to the debate over ADHD and its related medications. The authors do declare that they consider themselves critics of ADHD. The requests for access to Merzon, Manor, et al.'s dataset were therefore sent through a third neutral party. 


\section{References}

1. Merzon E, Manor I, Rotem A, et al. ADHD as a Risk Factor for Infection With Covid-19. Journal of Attention Disorders. 2020:1087054720943271.

2. Foreman DM. Attention deficit hyperactivity disorder: legal and ethical aspects. Archives of disease in childhood. 2006;91(2):192194.

3. Parrillo VN. Encyclopedia of social problems. Sage Publications; 2008.

4. Cortese S. Debate: are stimulant medications for attentiondeficit/hyperactivity disorder effective in the long term? Journal of the American Academy of Child and Adolescent Psychiatry. 2019;58(10):936.

5. American Psychiatric Association. Diagnostic and Statistical Manual of Mental Disorders (DSM-5®). American Psychiatric Pub; 2013.

6. Lange KW, Reichl S, Lange KM, Tucha L, Tucha O. The history of attention deficit hyperactivity disorder. ADHD Attention Deficit and Hyperactivity Disorders. 2010;2(4):241-255.

7. Visser SN, Danielson ML, Bitsko RH, et al. Trends in the parentreport of health care provider-diagnosed and medicated attentiondeficit/hyperactivity disorder: United States, 2003-2011. Journal of the American Academy of Child \& Adolescent Psychiatry. 2014;53(1):34-46.

8. Danielson ML, Bitsko RH, Ghandour RM, Holbrook JR, Kogan MD, Blumberg SJ. Prevalence of Parent-Reported ADHD Diagnosis and Associated Treatment Among U.S. Children and Adolescents, 2016. Journal of Clinical Child \& Adolescent Psychology. 2018;47(2):199-212.

9. Kooij JJS, Bijlenga D, Salerno L, et al. Updated European Consensus Statement on diagnosis and treatment of adult ADHD. European psychiatry. 2019;56:14-34.

10. Biederman J, Spencer T. Attention-deficit/hyperactivity disorder (ADHD) as a noradrenergic disorder. Biological psychiatry. 1999;46(9):1234-1242.

11. Sayal K, Prasad V, Daley D, Ford T, Coghill D. ADHD in children and young people: prevalence, care pathways, and service provision. The Lancet Psychiatry. 2018;5(2):175-186.

12. Barkley RA. Emotional dysregulation is a core component of ADHD. In: Barkley RA, ed. Attention-deficit hyperactivity disorder: A handbook for diagnosis and treatment. The Guilford Press; 2015:81-115.

13. Daley D. Attention deficit hyperactivity disorder: a review of the essential facts. Child: care, health and development. 2006;32(2):193-204
14. Sultan RS, Correll CU, Schoenbaum M, King M, Walkup JT, Olfson M. National patterns of commonly prescribed psychotropic medications to young people. Journal of child and adolescent psychopharmacology. 2018;28(3):158-165.

15. National Institute for Health and Care Excellence. Attention deficit hyperactivity disorder: diagnosis and management. NICE Guideline [NG87]. Available at: https://www.nice.org.uk/guidance/NG87. Accessed August 20, 2020. 2019.

16. Groenman AP, Schweren LJS, Dietrich A, Hoekstra PJ. An update on the safety of psychostimulants for the treatment of attention-deficit/hyperactivity disorder. Expert Opinion on Drug Safety. 2017;16(4):455-464.

17. Chang Z, Ghirardi L, Quinn PD, Asherson P, D’Onofrio BM, Larsson H. Risks and Benefits of Attention-Deficit/Hyperactivity Disorder Medication on Behavioral and Neuropsychiatric Outcomes: A Qualitative Review of Pharmacoepidemiology Studies Using Linked Prescription Databases. Biological psychiatry. 2019;86(5):335-343.

18. Storebø OJ, Pedersen N, Ramstad E, et al. Methylphenidate for attention deficit hyperactivity disorder (ADHD) in children and adolescents-assessment of adverse events in non-randomised studies. Cochrane Database of Systematic Reviews. 2018(5).

19. Boland H, DiSalvo M, Fried R, et al. A literature review and meta-analysis on the effects of ADHD medications on functional outcomes. Journal of Psychiatric Research. 2020;123:21-30.

20. Pollak Y, Poni B, Gershy N, Aran A. The role of parental monitoring in mediating the link between adolescent ADHD symptoms and risk-taking behavior. Journal of attention disorders. 2020;24(8):1141-1147.

21. Ruiz-Goikoetxea M, Cortese S, Aznarez-Sanado M, et al. Risk of unintentional injuries in children and adolescents with ADHD and the impact of ADHD medications: A systematic review and metaanalysis. Neuroscience \& Biobehavioral Reviews. 2018;84:63-71.

22. Thompson AL, Molina BSG, Pelham Jr W, Gnagy EM. Risky driving in adolescents and young adults with childhood ADHD. Journal of pediatric psychology. 2007;32(7):745-759.

23. Molina BSG, Howard AL, Swanson JM, et al. Substance use through adolescence into early adulthood after childhooddiagnosed ADHD: findings from the MTA longitudinal study. Journal of Child Psychology and Psychiatry. 2018;59(6):692-702.

24. Spiegel T, Pollak Y. Attention Deficit/Hyperactivity Disorder and Increased Engagement in Sexual Risk-Taking Behavior: The Role of Benefit Perception. Frontiers in Psychology. 2019;10(1043). 
25. Ginsberg Y, Hirvikoski T, Lindefors N. Attention Deficit Hyperactivity Disorder (ADHD) among longer-term prison inmates is a prevalent, persistent and disabling disorder. $B M C$ psychiatry. 2010;10:112-112.

26. Mohr-Jensen C, Müller Bisgaard C, Boldsen SK, Steinhausen HC. Attention-Deficit/Hyperactivity Disorder in Childhood and Adolescence and the Risk of Crime in Young Adulthood in a Danish Nationwide Study. Journal of the American Academy of Child \& Adolescent Psychiatry. 2019;58(4):443-452.

27. Huang K-L, Wei H-T, Hsu J-W, et al. Risk of suicide attempts in adolescents and young adults with attention-deficit hyperactivity disorder: a nationwide longitudinal study. The British Journal of Psychiatry. 2018;212(4):234-238.

28. Nigg JT. Attention-deficit/hyperactivity disorder and adverse health outcomes. Clinical psychology review. 2013;33(2):215228.

29. Chen VC-H, Yang Y-H, Liao Y-T, et al. The association between methylphenidate treatment and the risk for fracture among young ADHD patients: A nationwide population-based study in Taiwan. PloS one. 2017;12(3).

30. Chang Z, Lichtenstein P, Halldner L, et al. Stimulant ADHD medication and risk for substance abuse. Journal of Child Psychology and Psychiatry. 2014;55(8):878-885.

31. Liang SH-Y, Yang Y-H, Kuo T-Y, et al. Suicide risk reduction in youths with attention-deficit/hyperactivity disorder prescribed methylphenidate: a Taiwan nationwide population-based cohort study. Research in developmental disabilities. 2018;72:96-105.

32. Boutron I, Ravaud P. Misrepresentation and distortion of research in biomedical literature. Proceedings of the National Academy of Sciences. 2018;115(11):2613-2619.

33. Simmons JP, Nelson LD, Simonsohn U. False-positive psychology: Undisclosed flexibility in data collection and analysis allows presenting anything as significant. Psychological science. 2011;22(11):1359-1366.

34. Al-Marzouki S, Roberts I, Marshall T, Evans S. The effect of scientific misconduct on the results of clinical trials: a Delphi survey. Contemporary clinical trials. 2005;26(3):331-337.

35. Whitaker R, Cosgrove L. Psychiatry under the influence: Institutional corruption, social injury, and prescriptions for reform. Springer; 2015.

36. Qato DM, Alexander GC, Guadamuz JS, Lindau ST. Prescription Medication Use Among Children and Adolescents in the United States. Pediatrics. 2018;142(3):e20181042.
37. Chai G, Governale L, McMahon AW, Trinidad JP, Staffa J, Murphy D. Trends of Outpatient Prescription Drug Utilization in US Children, 2002-2010. Pediatrics. 2012;130(1):23.

38. Merzon E, Gutbir Y, Vinker S, et al. Early Childhood Shigellosis and Attention Deficit Hyperactivity Disorder: A PopulationBased Cohort Study with a Prolonged Follow-up. Journal of Attention Disorders. 2020:1087054720940392.

39. Merzon E, Tworowski D, Gorohovski A, et al. Low plasma 25 $(\mathrm{OH})$ vitamin D level is associated with increased risk of COVID19 infection: an Israeli population-based study. The FEBS Journal. 2020.

40. Mathieu S, Giraudeau B, Soubrier M, Ravaud P. Misleading abstract conclusions in randomized controlled trials in rheumatology: comparison of the abstract conclusions and the results section. Joint Bone Spine. 2012;79(3):262-267.

41. Altwairgi AK, Booth CM, Hopman WM, Baetz TD. Discordance between conclusions stated in the abstract and conclusions in the article: analysis of published randomized controlled trials of systemic therapy in lung cancer. Journal of clinical oncology. 2012;30(28):3552-3557.

42. Brown AW, Bohan Brown MM, Allison DB. Belief beyond the evidence: using the proposed effect of breakfast on obesity to show 2 practices that distort scientific evidence. The American journal of clinical nutrition. 2013;98(5):1298-1308.

43. Wolraich ML, Doffing MA. Pharmacokinetic Considerations in the Treatment of Attention-Deficit Hyperactivity Disorder with Methylphenidate. CNS Drugs. 2004;18(4):243-250.

44. Storebø OJ, Krogh HB, Ramstad E, et al. Methylphenidate for attention-deficit/hyperactivity disorder in children and adolescents: Cochrane systematic review with meta-analyses and trial sequential analyses of randomised clinical trials. Bmj. 2015;351.

45. Visser SN, Danielson ML, Wolraich ML, et al. Vital signs: national and state-specific patterns of attention deficit/hyperactivity disorder treatment among insured children aged 2-5 years-United States, 2008-2014. Morbidity and Mortality Weekly Report. 2016;65(17):443-450.

46. Evans WN, Morrill MS, Parente ST. Measuring inappropriate medical diagnosis and treatment in survey data: The case of ADHD among school-age children. Journal of health economics. 2010;29(5):657-673.

47. Halldner L, Tillander A, Lundholm C, et al. Relative immaturity and ADHD: findings from nationwide registers, parent-and selfreports. Journal of child psychology and psychiatry.

2014;55(8):897-904. 
48. Hoshen MB, Benis A, Keyes KM, Zoëga H. Stimulant use for $\mathrm{ADHD}$ and relative age in class among children in Israel. Pharmacoepidemiology and drug safety. 2016;25(6):652-660.

49. Gathercole SE. Cognitive approaches to the development of short-term memory. Trends in cognitive sciences. 1999;3(11):410-419.

50. Hoogman M, Muetzel R, Guimaraes JP, et al. Brain imaging of the cortex in ADHD: a coordinated analysis of large-scale clinical and population-based samples. American Journal of Psychiatry. 2019:appi-ajp.

51. Ludvigsson JF. Systematic review of COVID-19 in children shows milder cases and a better prognosis than adults. Acta Paediatrica. 2020;109(6):1088-1095.

52. Webb AR, Pilbeam C, Hanafin N, Holick MF. An evaluation of the relative contributions of exposure to sunlight and of diet to the circulating concentrations of 25-hydroxyvitamin D in an elderly nursing home population in Boston. The American journal of clinical nutrition. 1990;51(6):1075-1081.

53. Amour MDS, O'Leary DD, Cairney J, Wade TJ. What is the effect of ADHD stimulant medication on heart rate and blood pressure in a community sample of children? Canadian Journal of Public Health. 2018;109(3):395-400.

54. Cortese S, Coghill D, Santosh P, Hollis C, Simonoff E. Starting ADHD medications during the COVID-19 pandemic: recommendations from the European ADHD Guidelines Group. The Lancet Child \& Adolescent Health. 2020;4(6):e15.

55. Kousik SM, Napier TC, Carvey PM. The effects of psychostimulant drugs on blood brain barrier function and neuroinflammation. Frontiers in pharmacology. 2012;3:121-121.

56. Sajja RK, Rahman S, Cucullo L. Drugs of abuse and blood-brain barrier endothelial dysfunction: A focus on the role of oxidative stress. Journal of Cerebral Blood Flow \& Metabolism. 2016;36(3):539-554.

57. Steen RG. Retractions in the scientific literature: is the incidence of research fraud increasing? Journal of medical ethics. 2011;37(4):249-253.

58. Boutron I, Altman DG, Hopewell S, Vera-Badillo F, Tannock I, Ravaud P. Impact of spin in the abstracts of articles reporting results of randomized controlled trials in the field of cancer: the SPIIN randomized controlled trial. Journal of Clinical Oncology. 2014;32(36):4120-4126.

59. Boutron I, Haneef R, Yavchitz A, et al. Three randomized controlled trials evaluating the impact of "spin" in health news stories reporting studies of pharmacologic treatments on patients'/caregivers' interpretation of treatment benefit. $B M C$ medicine. 2019;17(1):105.

60. Stroebe W, Postmes T, Spears R. Scientific misconduct and the myth of self-correction in science. Perspectives on Psychological Science. 2012;7(6):670-688.

61. Cumming G. The New Statistics: Why and How. Psychological Science. 2013;25(1):7-29.

62. Schwarz A. ADHD nation: Children, doctors, big pharma, and the making of an American epidemic. Simon and Schuster; 2017.

63. Ophir Y. Evidence that ADHD is not purely a bio-medical disease. In. Findings from 3 consecutive studies are available by the authorin-preparation.

64. Whitaker R. Anatomy of an epidemic: Psychiatric drugs and the astonishing rise of mental illness in America. Ethical Human Psychology and Psychiatry. 2005;7(1):23.

65. Hadland SE, Cerdá M, Earlywine JJ, Krieger MS, Anderson TS, Marshall BDL. Analysis of Pharmaceutical Industry Marketing of Stimulants, 2014 Through 2018. JAMA Pediatrics. 2020;174(4):385-387.

66. DeJong C, Aguilar T, Tseng C-W, Lin GA, Boscardin WJ, Dudley RA. Pharmaceutical Industry-Sponsored Meals and Physician Prescribing Patterns for Medicare Beneficiaries. JAMA Internal Medicine. 2016;176(8):1114-1122.

67. Konrad-Bindl DS, Gresser U, Richartz BM. Changes in behavior as side effects in methylphenidate treatment: review of the literature. Neuropsychiatric disease and treatment. 2016;12:2635.

68. Faraone SV. The pharmacology of amphetamine and methylphenidate: Relevance to the neurobiology of attentiondeficit/hyperactivity disorder and other psychiatric comorbidities. Neuroscience \& Biobehavioral Reviews. 2018;87:255-270.

69. Whitely M. The rise and fall of ADHD child prescribing in Western Australia: Lessons and implications. Australian \& New Zealand Journal of Psychiatry. 2012;46(5):400-403.

70. Winterstein AG, Gerhard T, Shuster J, Johnson M, Zito JM, Saidi A. Cardiac safety of central nervous system stimulants in children and adolescents with attention-deficit/hyperactivity disorder. Pediatrics. 2007;120(6):e1494-e1501.

71. Dalsgaard S, Kvist AP, Leckman JF, Nielsen HS, Simonsen M. Cardiovascular safety of stimulants in children with attentiondeficit/hyperactivity disorder: a nationwide prospective cohort study. Journal of child and adolescent psychopharmacology. 2014;24(6):302-310.

72. Mosholder AD, Gelperin K, Hammad TA, Phelan K, JohannLiang R. Hallucinations and other psychotic symptoms associated 
with the use of attention-deficit/hyperactivity disorder drugs in children. Pediatrics. 2009;123(2):611-616.

73. Shyu Y-C, Yuan S-S, Lee S-Y, et al. Attentiondeficit/hyperactivity disorder, methylphenidate use and the risk of developing schizophrenia spectrum disorders: A nationwide population-based study in Taiwan. Schizophrenia Research. 2015;168(1):161-167.

74. DelBello MP, Soutullo CA, Hendricks W, Niemeier RT, McElroy SL, Strakowski SM. Prior stimulant treatment in adolescents with bipolar disorder: association with age at onset. Bipolar disorders. 2001;3(2):53-57.

75. Cherland E, Fitzpatrick R. Psychotic side effects of psychostimulants: a 5-year review. The Canadian Journal of Psychiatry. 1999;44(8):811-813.

76. Cressman AM, Macdonald EM, Huang A, et al. Prescription stimulant use and hospitalization for psychosis or mania: a population-based study. Journal of clinical psychopharmacology. 2015;35(6):667.

77. McCarthy S, Cranswick N, Potts L, Taylor E, Wong ICK. Mortality associated with attention-deficit hyperactivity disorder (ADHD) drug treatment. Drug safety. 2009;32(11):1089-1096.

78. Gould MS, Walsh BT, Munfakh JL, et al. Sudden Death and Use of Stimulant Medications in Youths. American Journal of Psychiatry. 2009;166(9):992-1001. 